D0I: 10.12957/demetra.2016.20414

\title{
Análise comparativa de guias alimentares: proximidades e distinções entre três países
}

\section{The comparative analysis of dietary guidelines: similarities and distinctions between three countries}

Ludmila Morais de Andrade'

Cláudia Boccal

${ }^{1}$ Universidade Federal do Estado do Rio de Janeiro, Escola de Nutrição. Rio de Janeiro-RJ, Brasil.

Correspondência / Correspondence Ludmila Morais de Andrade E-mail: lud.mandrade@hotmail.com

\section{Resumo}

A Organização Mundial de Saúde propõe que os governos forneçam informações à população para facilitar a adoção de escolhas alimentares mais saudáveis em uma linguagem que seja compreendida por todas as pessoas e que leve em conta questões culturais, sociais, econômicas e ambientais. Muitos países estão trabalhando na construção de seus próprios guias alimentares, com objetivos de aconselhamento nutricional e melhoria na qualidade de vida da população. No entanto, cada um deles possui características particulares que consideram o perfil alimentar e nutricional da população em questão, podendo conter recomendações gerais distintas entre si. A elaboração de guias alimentares abrange diversas etapas, sendo, portanto, um processo bastante complexo. Nesse sentido, o presente estudo analisou três guias alimentares atuais (Brasil, Estados Unidos e Portugal), com o intuito de identificar semelhanças e diferenças entre eles, buscando relacioná-los ao seu contexto de produção. Foi realizada revisão de literatura a fim de identificar estudos que tratassem do assunto e que trouxessem o contexto de produção desses documentos, sendo efetuada a leitura sistematizada de cada um dos guias, adotando a análise temática com base nas seguintes categorias analíticas: conteúdo abordado, estratégia didática adotada e tipo de linguagem utilizada para a população. Os guias alimentares analisados são muito distintos entre si. O guia português se mostra de forma mais sucinta, o que pode facilitar a leitura pela população. No entanto, não tem a riqueza de informações dos demais. $\mathrm{O}$ americano apresenta suas recomendações de forma mais técnica, enquanto o brasileiro tem o foco no alimento e não no nutriente, como o americano, enfatizando a importância das dimensões sociais e culturais nas práticas alimentares. 
Palavras-chave: Guia alimentar. Educação alimentar e nutricional. Saúde Pública.

\section{Abstract}

The World Health Organization suggests that governments provide information to the people in order to facilitate the adoption of healthier food choices in a language that is understood by everyone as well as taking into account the cultural, social, economic and environmental issues. Many countries are working in the formulation of their own dietary guidelines, with goals for nutritional counseling and improvement in people's quality of life. However, each one has particular characteristics when considering the eating and nutritional habits of the population concerned and it may contain various general recommendations to each other. The preparation of food guides includes several steps and is therefore a very complex process. In this context, the present study investigates three current dietary guidelines (Brazil, United States and Portugal), in order to identify similarities and differences between them, trying to relate them to their production environment. A literature review was conducted in order to identify studies that address to the issue and bring the context of production of these documents, and by making a systematic reading of each of the guides adopting the thematic analysis based on the following analytical categories: content addressed, teaching strategy adopted and type of language used for population. The analyzed dietary guidelines are very distinct from each other. The guide of Portugal shown more succinctly, which can facilitate the reading by the population. However, it does not have the wealth of information as the others. The US has its most technical form of recommendations, while the Brazilian one has focused on the food rather than on nutrient, such as the Americans, emphasizing the importance of social and cultural dimensions in eating habits.

Key words: Food guide. Food and nutrition education. Public Health.

\section{Introdução}

O comportamento alimentar humano sofre influência do estado fisiológico, do estado psicológico e das condições ambientais de cada indivíduo. Portanto, além das necessidades básicas do nosso organismo, fatores como localidade, renda, oferta ou escassez de determinados alimentos, 
regionalismo, tabus alimentares e crenças sociais, culturais e religiosas, vão interferir no consumo alimentar da população. ${ }^{1}$ Os diversos desafios contemporâneos relacionados à alimentação e nutrição, tanto a desnutrição ligada à fome e pobreza, como a obesidade relacionada ao estilo de vida e ao ambiente obesogênico da sociedade atual, entre outras questões (como o atual modelo de produção de alimentos, por exemplo), têm levado os países a determinar políticas e diretrizes nacionais voltadas ao estabelecimento de ações para o enfrentamento dessas questões. ${ }^{2}$

É obrigação do Estado respeitar, promover e proteger o direito à alimentação adequada, além de apresentar medidas para alcançar progressivamente a sua plena realização. ${ }^{3}$ Sob o enfoque de segurança alimentar e nutricional (SAN), cabe ao Estado proporcionar um ambiente econômico, social, político e cultural pacífico, estável e propício à alimentação adequada e saudável, no qual as pessoas possam se alimentar com liberdade e dignidade. ${ }^{4}$

Entretanto, o crescente número de informações e a diversidade de produtos divulgados pelos meios de comunicação podem dificultar a adoção e distanciar os consumidores das práticas alimentares saudáveis em função do apelo que tais produtos possuem e da própria dificuldade que os consumidores têm de diferenciar alimentos saudáveis e aqueles não saudáveis. Em pesquisa realizada pelo Ministério da Saúde, observou-se que 72,6\% das peças publicitárias de alimentos veiculadas na televisão aberta e por assinatura são de produtos com altos teores de gorduras, açúcares e sal pertencentes a cinco categorias de produtos, quais sejam: salgadinhos de pacote, bebidas gaseificadas, cereais matinais, fastfood e doces. ${ }^{5}$ Dessa forma, torna-se mais complexa a divulgação de mensagens nutricionais adequadas à população e, por este motivo, é de suma importância a criação de estratégias para orientação de práticas alimentares que tenham como objetivo informar a população sobre os malefícios de uma dieta pouco saudável e, ainda, como pôr em prática no dia a dia os benefícios que a alimentação mais salutar pode oferecer.

A OMS propõe que os governos forneçam informações à população para facilitar a adoção de escolhas alimentares mais saudáveis em uma linguagem que seja compreendida por todas as pessoas e que leve em conta questões culturais, sociais, econômicas e ambientais. ${ }^{6}$ Um guia alimentar é um instrumento oficial que define diretrizes alimentares como forma de orientação de escolhas de alimentos e hábitos mais saudáveis por uma determinada população, situando-se como uma medida de incentivo para a adoção de práticas alimentares sadias, visto que busca divulgar informações que possam apoiar as escolhas da população. ${ }^{7}$

O Departamento de Serviço Humano e Saúde (Departament of Health and Human Service - HHS) e o Departamento de Agricultura dos Estados Unidos (United States Departament of Agriculture - USDA) vêm publicando guias alimentares há algumas décadas. O primeiro guia alimentar de que se tem conhecimento foi proposto em 1916 nos Estados Unidos da América (EUA), por Caroline Hunt, que recomendou uma alimentação saudável por meio de mensagens sobre necessidades nutricionais e composição alimentar conhecidas à época. Outros guias alimentares 
foram publicados posteriormente baseados na proposta da autora, enfocando os problemas de cada época, como a seleção de alimentos durante a recessão econômica e alternativas alimentares durante a Segunda Guerra Mundial. Por volta de 1940, após as novas recomendações do National Research Council, de 1941, foi criado o guia alimentar popularmente chamado Basic Seven Food Guide, desenvolvido no período da Segunda Guerra Mundial, com o objetivo de sugerir substituições alimentares, em caso de escassez de comida, e recomendar alimentos econômicos durante a recessão. No entanto, foi considerado muito complexo, sendo também criticada a falta de indicação de como substituir quantitativamente os alimentos, pela ausência de informação sobre as porções alimentares. ${ }^{8}$ Percebe-se, portanto, que o enfoque do guia Basic Seven Food Guide tinha íntima relação com o contexto político da época.

No final de 1940, dois estudos, o Twin Cities e o Framingham, começaram a examinar a associação entre fatores dietéticos e o aumento do risco de doenças crônicas não transmissíveis. Por volta de 1950, reconheceu-se que a saúde não é simplesmente ausência de doença e, para garantir o bem-estar, eram necessárias escolhas alimentares adequadas e estilo de vida saudável para a promoção da saúde. Diante desse contexto, em 1956, especialistas em nutrição publicaram um novo guia alimentar, o Basic Four, com o número de porções recomendadas dos quatro grupos de alimentos, sendo eles: leite, carnes, vegetais e frutas e pães e cereais. ${ }^{9}$

A partir da década de 1970, pesquisadores associaram o alto consumo de certos alimentos com o aparecimento de determinadas doenças. Em 1979, foi publicado o Hassle Free Guide, no qual foi incluído o quinto grupo alimentar (gorduras, açúcares, álcool), chamando a atenção para o consumo moderado desses alimentos. Em 1980, após estudo realizado pela Sociedade Americana de Nutrição Clínica, que verificou uma forte associação entre alimentação e saúde, USDA e HHS publicaram a primeira edição do Guia Alimentar para Americanos, tendo como objetivo o enfoque na saúde, com base no padrão dietético e em dados de consumo e de composição dos alimentos, bem como na utilidade do próprio Guia para o consumidor. Em 1990, o Congresso aprovou o Monitoramento Nacional de Nutrição, que torna obrigatório revisar, atualizar e publicar as orientações dietéticas a cada cinco anos. Desde então, esses dois departamentos vêm realizando revisões dos guias alimentares publicados no país. ${ }^{10}$

Na Conferência Internacional de Nutrição, realizada em Roma, em 1992, foram identificadas estratégias e ações para melhorar o consumo alimentar e o bem-estar nutricional da população. De acordo com esse evento, torna-se importante estimular a elaboração de guias alimentares para diferentes grupos etários e, para atingir este objetivo, cada país deve planejar ações de acordo com sua cultura e com os problemas de saúde relacionados à alimentação. ${ }^{11}$ Posteriormente, em 1995, a FAO e a OMS realizaram uma conferência articulada, traçando diretrizes para o desenvolvimento dos guias alimentares. O conteúdo dessas diretrizes reforçava que o principal fator para desenvolver um guia alimentar é a identificação de problemas referentes à alimentação. A partir desse momento, alguns países decidiram utilizar como representação gráfica o formato do guia alimentar seguindo 
o modelo norte-americano (com o formato de pirâmide), com algumas exceções como Canadá (que desenvolveu o arco-íris), Costa Rica (com o formato de uma pizza), Guatemala (que optou pela representação gráfica de um pote de cerâmica) e o México (que adotou a figura da maçã). Já os países da Europa optaram pela pirâmide e pelo círculo. ${ }^{12}$

Desde então, diversos países vêm se esforçando na construção de seus próprios guias alimentares, com objetivos semelhantes no que tange ao aconselhamento nutricional de indivíduos e populações e à melhoria na qualidade de vida. No entanto, cada um deles possui características particulares que consideram não apenas o perfil alimentar e nutricional da população em questão, bem como questões sociais, culturais, econômicas e políticas específicas locais, podendo conter recomendações gerais distintas entre si. A elaboração de guias alimentares abrange diversas etapas, sendo, portanto, um processo bastante complexo. Neste contexto, o presente estudo busca analisar três guias alimentares atuais de diferentes países, com o intuito de identificar semelhanças e diferenças entre eles, buscando relacioná-los ao seu processo de elaboração.

\section{Metodologia}

O estudo realizado utilizou como base primária de pesquisa os guias alimentares voltados para população acima de dois anos de idade, presentes na biblioteca da homepage da Rede de Alimentação e Nutrição do Sistema Único de Saúde (Redenutri). Entre eles, foram escolhidos o guia dos EUA (2010), por ser o primeiro país a propor um guia alimentar para a sua população, o de Portugal (2003), a fim de representar a Europa, e a nova versão do guia alimentar brasileiro publicada em 2014. Após esse levantamento bibliográfico, foi feita revisão de literatura para identificar estudos que tratassem da temática, bem como possíveis trabalhos que comparassem guias alimentares de diferentes países. Além disso, foram identificados trabalhos que trouxessem o contexto de produção desses documentos.

Posteriormente, foi realizada a leitura de cada um dos guias alimentares dos três países escolhidos, sendo feita uma sistematização do material com o intuito de efetuar a análise documental. Segundo Rampazzo, ${ }^{13}$

A análise documental consiste em uma série de operações que visam estudar e analisar um ou vários documentos para descobrir as circunstâncias sociais e econômicas com as quais podem estar relacionados.

Dessa forma, a análise temática foi realizada com base na construção das seguintes categorias analíticas, que foram transversais à apreciação dos documentos: tamanho, conteúdo abordado, estratégia didática adotada e tipo de linguagem utilizada para a população. A revisão de literatura e análise do conteúdo dos guias ocorreu no período entre junho e dezembro de 2015. 


\section{Resultados e Discussão}

\section{O pioneirismo do guia alimentar americano}

O processo para criar cada edição do guia alimentar americano é um esforço conjunto do USDA e do HHS e inclui três fases. Na primeira etapa, um Comitê Consultivo de Orientações Dietéticas (DGAC) é nomeado para conduzir uma análise dos novos dados científicos e informações sobre alimentação e saúde, além de preparar um relatório que resume as suas conclusões. A análise do Comitê é o principal recurso para os dois departamentos para o desenvolvimento das diretrizes dietéticas para os americanos. Em relação ao guia americano atual, a análise do Comitê foi concluída em junho de 2010, sendo o relatório disponibilizado aos órgãos públicos federais. Durante a segunda fase, USDA e HHS desenvolvem o documento Guia de Orientações Dietéticas para Americanos". As audiências para apreciação desse documento incluem como públicoalvo políticos, educadores físicos, profissionais da área de alimentação e nutrição e prestadores de cuidados à saúde. Semelhante às edições anteriores, a edição de 2010 baseia-se no relatório do Comitê Consultivo, considerando os comentários dos órgãos públicos federais. As evidências científicas pautam a elaboração das diretrizes e o desenvolvimento de políticas. Na terceira e última etapa, os dois departamentos desenvolvem mensagens e materiais que comunicam o guia para o público em geral. ${ }^{14}$

As orientações dietéticas americanas (Dietary Guide lines) fornecem recomendações alimentares baseadas em evidências para os americanos maiores de dois anos de idade. Essas recomendações visam a promover a saúde, prevenir doenças crônicas e ajudar as pessoas a atingir e manter um peso saudável. Os órgãos públicos de saúde, prestadores de cuidados de saúde e instituições de ensino dependem dessas recomendações e estratégias das diretrizes dietéticas. Portanto, essas orientações podem ter impacto significativo sobre as ações de alimentação e nutrição nos Estados Unidos, pois constituem a base de programas de nutrição e orientam iniciativas de prevenção de doenças e promoção da saúde. ${ }^{14}$

O guia americano atual começa com uma introdução pautada na história e no desenvolvimento dos guias alimentares dos EUA. Nessa introdução são explicados os termos utilizados ao longo do documento para facilitar a compreensão dos leitores. E explica, ainda, a sua importância para a promoção da saúde e prevenção de doenças, bem como os principais usos do guia: desenvolvimento de materiais educativos e programas governamentais relacionados à alimentação e nutrição. Esse

guia de 2010 apresenta os números referentes aos casos de doenças crônicas relacionadas com a alimentação, como doenças cardiovasculares (atingindo $37 \%$ da população americana), hipertensão arterial (34\% da população adulta), diabetes (11\% dos americanos), câncer (41\% da população será 
diagnosticada com câncer durante a vida) e osteoporose (uma em cada duas mulheres e um em cada quatro homens maior de 50 anos terá uma fratura relacionada à osteoporose em sua vida), enfatizando as principais causas dessas doenças e os riscos para a saúde. ${ }^{14}$

Baseado nos dados sobre as doenças crônicas não transmissíveis prevalentes nos EUA, e com o crescimento do consumo alimentar dos americanos, especialmente de alimentos ultraprocessados, os quais acarretam aumento do sobrepeso e obesidade no país, o guia aborda no segundo capítulo recomendações sobre balanço calórico para manutenção do peso e controle da ingestão. Nesse capítulo, são listados os 25 alimentos mais consumidos pelos americanos nas diferentes faixas etárias, em que é possível perceber a presença massiva de produtos industrializados, doces, bebidas açucaradas, além de bebidas alcoólicas. É apresentada, ainda, uma comparação entre os anos 1970 e 2008, mostrando o aumento da prevalência de obesidade no país (de $15 \%$ para $34 \%$ entre adultos).$^{14}$ Como ferramentas de auxílio, é demonstrada a forma de cálculo do Índice de Massa Corporal (IMC), para avaliação de adequação de peso, e as estimativas de calorias necessárias de acordo com sexo, idade e prática de atividade física, explicando como cada macronutriente (proteína, carboidrato, lipídio), além do álcool, contribuem para o cálculo dessas calorias.

No terceiro capítulo, destaca-se a importância da redução de certos alimentos e nutrientes (sódio, gordura saturada, trans, colesterol, açúcar e álcool) responsáveis pelo incremento das doenças citadas na introdução do guia. Dando sequência, o quarto capítulo traz os alimentos e nutrientes que devem ser acrescentados na dieta e quais as funções deles no organismo. São citados ainda nutrientes adicionais, como suplementos, em algumas fases da vida, como a gestação, amamentação ou para pessoas que fazem dietas restritas. Por conseguinte, o quinto capítulo trata da construção de padrões alimentares saudáveis, citando a alimentação vegetariana, dietas específicas de prevenção de doenças e para pessoas com alergias ou intolerâncias. Descreve também a importância da Segurança Alimentar e Nutricional, explicando processos de limpeza, separação, cozimento e armazenamento adequado dos alimentos.

Para finalizar, o guia aborda, no sexto e último capítulo, formas de ajudar as pessoas a fazerem escolhas mais salutares. Para tal, mostra que essas escolhas dependem de fatores individuais (idade, sexo, condição socioeconômica, genética, atividade física, entre outros), configurações do ambiente em que vive, atores políticos de influência (governo, agricultura e indústria), além das normas e valores sociais e culturais. Dessa forma,o guia faz um apelo à ação dos americanos, incluindo três princípios norteadores: assegurar que todos os americanos tenham acesso a alimentos nutritivos e oportunidades de praticar atividade física; facilitar a mudança no comportamento individual através de estratégias do ambiente e definir o cenário para uma alimentação saudável ao longo da vida, atividade física e manutenção do peso. 


\section{Portugal, representante europeu}

A Roda dos Alimentos, elaborada em Portugal no final da década de 1970, representada graficamente por um círculo subdividido em fatias de diferentes tamanhos que apresentava os alimentos com propriedades nutricionais semelhantes, foi um instrumento inovador utilizado por diversos países como material educativo. Teve como objetivo transformar informações nutricionais complexas em conceitos simples e fáceis de serem empregados pela população leiga. O prato é um importante símbolo da cultura portuguesa, pois a mesa é um lugar comum e fundamental para as refeições. De acordo com o guia português, os grupos de alimentos apresentados no formato do círculo mostram a mesma importância e visualmente se complementam, ao contrário da pirâmide, que representa a ideia hierarquizada dos grupos alimentares. ${ }^{15}$

Em 2003, foi desenvolvido o novo guia alimentar português elaborado em nove etapas: $1^{a}$ ) obtenção da opinião de pesquisadores sobre alimentação e nutrição; $2^{2}$ ) estabelecimento dos objetivos nutricionais, isto é, a recomendação de energia de 13 grupos populacionais de ambos os sexos (crianças acima de um ano até adultos), a distribuição de macronutrientes, micronutrientes e de fibras alimentares; $3^{\mathfrak{a}}$ ) definição dos sete grupos e 21 subgrupos de alimentos de acordo com a composição nutricional, os hábitos alimentares dos portugueses em substituição aos cinco grupos já utilizados na primeira versão da roda de alimentos. ${ }^{15}$

A 4aㅡ etapa do guia português foi o estabelecimento da porção padrão de cada grupo alimentar, baseada na média de peso de medidas caseiras ou em unidades. Na $5^{\mathbf{a}}$ etapa estabeleceu-se a porção de equivalente de cada grupo alimentar e na etapa seguinte definiu-se o número recomendado de porções diárias de cada grupo, com base em três dietas calculadas de acordo com o valor energético total de 1.300, 2.200 e 3.000 calorias. Na 7̣a etapa foi analisado se as três dietas ofertavam em quantidades adequadas os macro e micronutrientes de forma a atender às recomendações nutricionais. Somente os valores de sódio e iodo não atenderam. A 8ª etapa consistiu na apresentação dos resultados, e o ícone escolhido foi o formato do círculo por estar associado à imagem de um prato e por já ser reconhecido pelos indivíduos por conta da primeira versão do guia alimentar. ${ }^{15}$

Com relação ao perfil epidemiológico de Portugal, a hipertensão é a doença mais prevalente, e as doenças cardiovasculares e o câncer encontram-se entre as principais causas de morte. ${ }^{16} \mathrm{~A}$ proporção de pessoas com desnutrição é inferior a 2,5\%, ${ }^{17}$ em contrapartida, verifica-se prevalência de excesso de peso em 31,5\% das crianças de 7 a 9 anos, ${ }^{18}$ em $54 \%$ dos homens e em 46, \% das mulheres. ${ }^{19} \mathrm{O}$ atual padrão de alimentação em Portugal caracteriza-se pelo afastamento do modelo mediterrâneo, sendo observado crescimento no consumo de sódio e gorduras e redução de hortaliças e frutas, especialmente nas faixas etárias mais jovens. O consumo de bebidas alcoólicas permanece elevado, porém houve mudança para o tipo de bebida, com a diminuição no consumo de vinho e aumento de cerveja e de outras bebidas. ${ }^{20}$ 
Baseado nesse padrão alimentar e na prevalência da hipertensão, bem como no aumento de pessoas com sobrepeso e obesidade no país, o guia alimentar para a população portuguesa tem como foco a apresentação de sete grupos de alimentos e suas respectivas porções, enfatizando ainda limites de ingestão diária de sódio, cafeína e álcool, além de alertar para a leitura cuidadosa dos rótulos de produtos industrializados açucarados e salgados. Recomenda, ainda, a substituição do sal de cozinha por ervas aromáticas e especiarias na preparação de alimentos. Como forma de auxílio, exemplifica o tamanho das colheres, copos e xícaras, facilitando a compreensão das porções recomendadas na Roda. Cita o Índice de Massa Corporal (IMC) como medida de avaliação de adequação de peso e fala sobre a importância em manter um peso saudável, o qual pode ser alcançado por meio de atividade física regular e das recomendações previstas na Roda de Alimentos.

Por possuir apenas cinco páginas, comparando com as mais de 100 páginas dos guias americano e brasileiro, o material se apresenta de forma mais sucinta e direta. Dessa forma, pode atingir de modo mais fácil a população em questão, pois textos muito grandes ou com linguagem mais técnica não são atrativos ou de fácil compreensão. Por outro lado, quando informações relacionadas à alimentação e nutrição são resumidas, podem surgir dúvidas e dificuldades de entendimento dos leitores, podendo causar interpretações errôneas e inesperadas sobre o conteúdo que se deseja transmitir. Além de as recomendações serem passadas através de mensagens impositivas, diferentemente dos outros dois guias analisados.

Segundo Rodrigues et al., em 2006, ${ }^{15}$ apesar da falta de clareza a respeito do processo de desenvolvimento no guia anterior, observa-se que as porções recomendadas de cada grupo alimentar na nova Roda de Alimentos não diferem muito daquelas indicadas no guia alimentar anterior, desenvolvido em 1977. Rodrigues et al. ${ }^{15}$ relataram a necessidade de atualização das recomendações de energia e nutrientes para a população portuguesa, pois esses dados são de 1982. Além disso, as tabelas de composição química dos alimentos foram publicadas em 1985. Outro problema e, provavelmente, o mais relevante, deve-se ao fato de que a última pesquisa de consumo alimentar em Portugal foi realizada em 1980, o que pode não retratar o atual padrão dietético da população.

\section{O guia alimentar brasileiro como referência}

A transição epidemiológica no Brasil pode ser representada pela menor proporção de déficit de peso e de estatura e aumento do excesso de peso. Dados recentes da Pesquisa Nacional de Saúde (2014) apontam que, em 2013, 56,9\% dos adultos com idade superior a 18 anos apresentam excesso de peso e $20,8 \%$ já se encontram na faixa de obesidade, afetando especialmente as mulheres, que apresentam prevalências de excesso de peso e de obesidade maiores que as dos homens. ${ }^{21}$ 
Tal perfil nutricional, em parte, decorre das mudanças nos padrões de consumo alimentar. Verificou-se elevação, entre 2002 e 2008, de 30\% no número de refeições realizadas fora do domicílio. Também houve aumento na aquisição de alimentos pré-prontos (37,0\%), de refrigerantes à base de cola $(20,0 \%)$ e cerveja $(88,0 \%)$. Em contrapartida, diminuiu-se a aquisição de leguminosas $(19,4 \%)$ e cereais (20,5\%). ${ }^{22} \mathrm{O}$ crescimento do consumo de alimentos ultraprocessados (como embutidos, biscoitos recheados, refrigerantes, macarrão instantâneo, entre outros) tem sido considerado, pela Organização Mundial de Saúde (OMS), um dos principais fatores que vem contribuindo para a epidemia global de obesidade, diabetes e outras doenças crônicas não transmissíveis. ${ }^{23}$ Martins et al., em 2013, ${ }^{24}$ revelam, com base nos resultados das Pesquisas de Orçamentos Familiares, que o consumo de produtos ultraprocessados aumentou no período de 2002-2003 (20,8\%) a 2008-2009 (25,4\%) em todos os estratos de renda, com redução na participação de alimentos e ingredientes culinários.

Em 2006, devido à a situação de transição epidemiológica e nutricional, nas evidências científicas e na responsabilidade do governo em promover a saúde, direito de todo cidadão, o guia alimentar para a população brasileira é elaborado com o objetivo de contribuir para a orientação de práticas alimentares adequadas, buscando promoção da saúde e prevenção de doenças relacionadas à alimentação, e para sua implantação foi distribuída uma versão de bolso para a população. ${ }^{25}$

A OMS recomenda a atualização periódica das recomendações sobre alimentação adequada e saudável. A partir de 2011, portanto, o Ministério da Saúde, em parceria com o Núcleo de Pesquisas Epidemiológicas em Nutrição e Saúde da Universidade de São Paulo (Nupens/USP) e com o apoio da Organização Pan-Americana da Saúde (Opas/Brasil), iniciou o processo de elaboração de uma nova edição do Guia Alimentar para a População Brasileira. Para promover a construção coletiva e proporcionar a ampla participação e discussão do conteúdo desse documento, a Coordenação Geral de Alimentação e Nutrição (CGAN) do Ministério da Saúde organizou seis grandes etapas, que contribuíram para a versão final da nova edição do guia. ${ }^{6}$

A primeira etapa ocorreu em novembro de 2011 e foi realizada uma oficina de escuta com participantes de todo o Brasil. Estiveram presentes profissionais dos setores da saúde, educação, assistência social e agricultura, professores de universidades, dirigentes de conselhos profissionais e de associações profissionais e membros de organizações de controle social de políticas públicas e de defesa do consumidor. As discussões dos grupos e reuniões foram relatadas em uma plenária final da oficina com exposição dos diferentes pontos de vista e observações resultantes dos debates em grupo. Os resultados dessa oficina orientaram a primeira versão da nova edição do guia alimentar. ${ }^{6}$

Na segunda etapa foi elaborada a primeira versão da nova edição do guia, entre novembro de 2011 e julho de 2013, por uma equipe integrada por técnicos da CGAN/MS e da Opas e pesquisadores do Nupens/USP. Na terceira fase, avaliou-se a primeira versão da nova edição do guia alimentar em uma segunda oficina, realizada em agosto de 2013. As discussões dos grupos foram relatadas e discutidas em uma plenária final. Os resultados dessa oficina orientaram a 
elaboração de uma segunda versão da nova edição do guia alimentar, correspondendo à quarta etapa do processo, que correu entre setembro e dezembro de 2013, com a mesma equipe. ${ }^{6}$

Na quinta etapa, após avaliação e aprovação pelo Ministério da Saúde, a segunda versão da nova edição foi posta em consulta pública de 10 de fevereiro até 7 de maio de 2014. Durante esse período, foram realizadas diversas reuniões e grupos, buscando incentivar a discussão do conteúdo do guia, acolher as diferentes opiniões, estimular a divulgação da consulta em outros espaços de diálogo e incentivar contribuições e possíveis sugestões, contando com a participação de profissionais de saúde da rede de atenção ao SUS, gestores, profissionais da educação e assistência social e representantes da sociedade civil. Foram recebidas, no total, 3.125 contribuições de 436 indivíduos ou instituições. Com base nesse compilado de contribuições, iniciou-se a última etapa de elaboração da nova edição do guia alimentar. Tendo em vista a diversidade regional e os diferentes grupos populacionais a quem este guia se destina, o Ministério da Saúde pretende desenvolver outras estratégias de comunicação para divulgar o conteúdo do documento, como manuais, folhetos e vídeos. ${ }^{6}$

O guia brasileiro atual traz uma forte valorização da culinária do país, incentivando o consumo de alimentos regionais nas preparações culinárias. Além disso, em função do referencial da SAN, a preocupação com a sustentabilidade do ambiente está presente no guia brasileiro. Vale ressaltar que o atual conceito de SAN vigente no Brasil valoriza o acesso quanti e qualitativo aos alimentos sem comprometimento de outras necessidades humanas básicas, conforme o estabelecido pela Lei Orgânica de SAN de 2006:26

A segurança alimentar e nutricional consiste na realização do direito de todos ao acesso regular e permanente a alimentos de qualidade, em quantidade suficiente, sem comprometer o acesso a outras necessidades essenciais, tendo como base práticas alimentares promotoras de saúde que respeitem a diversidade cultural e que sejam ambiental, cultural, econômica e socialmente sustentáveis.

Percebe-se que os demais guias não trazem essas questões como fio condutor da sua orientação para a população. No caso do americano, a ideia de Segurança Alimentar aparece mais ligada à segurança sanitária do alimento.

O guia brasileiro apresenta, no primeiro capítulo, os princípios nos quais essa nova versão se fundamentou. Explica que suas recomendações são baseadas em estudos experimentais, clínicos, populacionais e antropológicos. No entanto, destaca que a alimentação é mais que a ingestão de nutrientes, logo, devemos levar em consideração as dimensões sociais e culturais nas práticas alimentares, além de aspectos mais abrangentes, como o impacto ambiental que nossa alimentação tem, enfatizando a importância de alimentos social e ambientalmente sustentáveis. Ainda nesse capítulo, sugere que as recomendações estejam em sintonia com o seu tempo, levando em conta o cenário da evolução da alimentação no país e as condições de saúde da população. 
No segundo capítulo, o guia aborda as recomendações gerais nas escolhas alimentares, baseando essas escolhas nas seguintes categorias: alimentos in natura ou minimamente processados; óleos, gorduras, sal e açúcar; alimentos processados e ultraprocessados, tendo como regras gerais: incentivar o consumo majoritário de alimentos in natura ou minimamente processados, sendo estes a base da alimentação; utilizar óleos, gorduras, açúcares e sal com moderação ao temperar e cozinhar alimentos e limitar a ingestão de produtos alimentícios prontos para consumo, evitandoos ou consumindo-os em pequenas quantidades como parte de refeições. E, ainda, como regra de ouro: preferir sempre alimentos e preparações culinárias a produtos prontos para consumo e evitar produtos ultraprocessados. Como didática, o guia, além de explicar cada categoria, oferta alguns exemplos de alimentos em cada uma.

O terceiro capítulo, intitulado "Dos Alimentos à Refeição", mostra como combinar alimentos na forma de refeições, valorizando as preparações culinárias consumidas nas diferentes regiões do país. São fornecidos exemplos com fotos de café da manhã, almoço, jantar e pequenas refeições, trabalhando sempre com o conceito de variedade e incentivando preparações culinárias. Nesse capítulo também é abordada a importância da conservação adequada dos alimentos e a forma de manipulação, preparo e higienização dos mesmos.

No capítulo seguinte, a atenção vai para o ato de comer, em que se deve levar em consideração o tempo, o espaço e a companhia em prol do prazer que a alimentação deve proporcionar, destacando três recomendações principais ao comer: regularidade e atenção, ambiente apropriado e comer em companhia. As fotos apresentadas nessa parte do guia nos remetem claramente à ideia de comensalidade. Para finalizar, o último capítulo oferece estratégias para facilitar a adesão das recomendações anteriores, mostrando os principais obstáculos enfrentados: informação, oferta, custo, habilidades culinárias, tempo e publicidade. Ao longo dos cinco capítulos, o guia nos incentiva à reflexão e senso crítico, mostrando a importância da autonomia nas escolhas alimentares, com foco nas combinações dos alimentos (considerando as diferenças regionais), no prazer e nas preparações culinárias e não nos nutrientes apenas.

\section{Comparando os guias alimentares}

Em relação ao tamanho, o guia português é o mais sucinto dentre os três, o que pode facilitar a leitura pela população. Como já sinalizado, o guia brasileiro de 2006 também apresentava uma versão sintética (guia de bolso), voltada à população. Dessa forma, como estratégia de comunicação social, seria interessante que tais guias apresentassem versões menores de forma a despertar o interesse e facilitar a divulgação das recomendações. Além disso, o guia brasileiro, apesar das suas 150 páginas contra as 100 do americano, possui letras maiores e mais figuras, o que torna a leitura mais dinâmica e menos cansativa. 
Quanto ao conteúdo abordado, o guia alimentar americano tem um enfoque maior em nutrientes quando comparado aos guias português e brasileiro, ambos com recomendações voltadas para os alimentos. No entanto, o guia português aborda especialmente os grupos alimentares e suas porções de alimentos, enquanto a versão atual do brasileiro não traz essa orientação. Essa questão das porções foi alvo de críticas durante o processo de elaboração do guia alimentar brasileiro porque, se, por um lado, havia uma defesa por parte de profissionais diversos da necessidade de incorporar a orientação de porções de alimentos (por uma preocupação em estabelecer recomendações quantitativas de alimentos); por outro, havia uma crítica de que a estratégia das porções não era de fácil compreensão.

Algumas questões relacionadas à alimentação como, por exemplo, quando, por que, onde e com quem as refeições são consumidas, que, em geral, não estão incluídas em guias alimentares, estão presentes na versão brasileira. Todas essas circunstâncias influenciam que tipo de alimento é consumido e em que quantidades, a sua digestão e absorção e o prazer em comer. ${ }^{27}$ Dieta também inclui os valores simbólicos e emocionais de alimentos, pratos e refeições, os quais contribuem para o prazer de comer, a construção de memórias e costumes, bem como para o reforço de relações e conexões, que são aspectos de saúde e bem-estar. ${ }^{28} \mathrm{O}$ novo guia alimentar brasileiro tenta resgatar esses valores e os coloca no mesmo plano das demais orientações. Para isso, ressalta a importância da culinária e comensalidade, em que se deve comer em companhia, em ambientes apropriados, com regularidade e atenção, contribuindo para o prazer da alimentação, sendo este um diferencial para os demais guias, que não abordam essas questões.

No tocante ao tipo de linguagem adotada em cada guia, o americano, por apresentar uma linguagem mais técnica, parece mais voltado a profissionais de saúde que à população em geral. O guia alimentar brasileiro de 2006 trazia como princípio o referencial positivo de suas recomendações, ou seja, enfatizando as vantagens e benefícios de uma alimentação saudável mais do que proibindo ou condenando práticas alimentares consideradas não sadias. Isso é mantido no guia brasileiro atual, que adota expressões como "evite", "limite" e "prefira", assim como o americano, que utiliza "escolha”, "acrescente", “consuma” e "substitua” para aconselhar hábitos mais salutares. Nesse contexto, o guia português apresenta mensagens impositivas para transmissão das suas recomendações, como "deve ser" ou "não deve ser" consumido determinado alimento, diferenciando-se dos demais.

Em relação às estratégias didáticas, os guias brasileiro e americano trazem orientações-chave para facilitar a compreensão e direcionar o leitor aos principais pontos abordados nos guias. O português, por trabalhar com a ideia de porções de alimentos, utiliza imagens de tamanhos de medidas caseiras, como colheres, copos e xícaras, facilitando a identificação das porções recomendadas. 


\section{Conclusão}

O processo de construção de um guia alimentar, independente do país, é complexo e abrange diversas etapas até a sua implantação e divulgação. Os guias alimentares analisados são muito distintos entre si. A ideia deste trabalho não era identificar qual seria o melhor guia alimentar visto que, como anteriormente apontado, o processo de elaboração de cada guia envolve questões sociais, culturais, econômicas, políticas, entre outras, próprias de cada contexto. No entanto, vale ressaltar que o guia alimentar brasileiro tem sido considerado uma importante referência entre os guias alimentares atuais, por valorizar questões como comer em companhia, diversidade cultural e, especialmente, o posicionamento político que defende ao recomendar o uso limitado de alimentos ultraprocessados, levando em conta as evidências científicas atuais.

\section{Referências}

1. Castro JM, Plunkett SS. How genes control real world intake: Palatability intake relationships. Nutrition 2001; 17(3):266-268.

2. Brasil. Ministério da Saúde. Política Nacional de Alimentação e Nutrição. Brasília: Ministério da Saúde; 2003.

3. World Health Organization. European action plan for food and nutrition policy 2007-2012. Copenhagen: WHO; 2008.

4. Vieira VL, Gregório MJ, Cervato-Mancuso AM, Graça APSR. Ações de alimentação e nutrição e sua interface com segurança alimentar e nutricional: uma comparação entre Brasil e Portugal. Saúde Soc 2013; 22(2):603-617.

5. Monteiro RA, Recine EG, Coutinho JG. Monitoração de propaganda de alimentos visando à prática da alimentação saudável. Brasília: Universidade de Brasília. Observatório de Políticas de Segurança Alimentar e Nutrição; 2008.

6. Brasil. Ministério da Saúde. Secretaria de Atenção à Saúde. Departamento de Atenção Básica. Guia alimentar para a população brasileira. 2 ed. Brasília: Ministério da Saúde; 2014.

7. Castro IRR, Castro LMC, Gugelmim A. Ações Educativas, programas e políticas envolvidos nas mudanças alimentares. In: Diez-Garcia RW, Cervato-Mancuso AM. Mudanças alimentares e educação nutricional. Rio de Janeiro: Guanabara Koogan; 2012. p. 18-34.

8. Myers EF, Britten P, Davis CA. Past, present, and future of the food guide pyramid. J Am Diet Assoc. 2001; 101(8):881-5.

9. Davis CA, Escobar A, Marcoe KL, Tarone C, Shaw A, Saltos S, et al. Food guide pyramid for young children 2 to 6 years old: Technical Report on Background and Development. Washington, DC: Center for Nutrition Policy and Promotion; 1999. 
10. Schneeman BO. Evolution of dietary guidelines. J Am Diet Assoc. 2003; 103(12):5-9.

11. World Health Organization. Food and Nutrition Board. International Conference on Nutrition. World Declaration and Plan of Action for Nutrition; dec. 1992; Rome. Geneva: WHO; 1992.

12. Hunt P, Rayner M, Gatenby S. A national food guide for UK. Background and development. Journal of Human Nutrition Dietetics 1995; 8(5):315-22.

13. Rampazzo SE, Corrêa, FZM. Desmistificando a metodologia científica. Erechim: Habilis; 2008.

14. U.S. Department of Agriculture. Dietary guidelines for Americans, 2010. 7th Edition. Washington, DC: U.S. Department of Health and Human Services; 2010.

15. Rodrigues SSP, Franchini B, Graça P, Almeida MDV. A new food guide for the Portuguese population: development and technical considerations. J Nutr Educ Behav. 2006; 38(3):189-95.

16. Instituto Nacional de Saúde Dr. Ricardo Jorge. Observatório Nacional de Saúde - ONSA. $4^{\circ}$ Inquérito Nacional de Saúde: 2005/2006. Lisboa: INSA; 2009.

17. Programa das Nações Unidas para o Desenvolvimento. Relatório de desenvolvimento humano 2007/2008: combater as alterações climáticas: solidariedade humana num mundo dividido. New York: PNUD; 2008.

18. Padez C, Fernandes T, Mourão I, Moreira P, Rosado V. Prevalence of overweight and obesity in 7-9 year-old portuguese children: trends in body mass index from 1970-2002. Am J Hum Biol. 2004; 16(6):670-678.

19. Carmo I, Santos S, Camolas J, Vieira J, Carreira M, Medina L, et al. Prevalence of obesity in Portugal. Obesity Reviews 2006; 7(3):233-237.

20. Willett WC, Sacks F, Trichopoulou A, Drescher G, Ferro-Luzzi A, Helsing E, et al. Mediterranean diet pyramid: a cultural model for healthy eating. Am J Clin Nutr. 1995; 61(6 Supl.):1403S-1406S.

21. Instituto Brasileiro de Geografia e Estatística. Pesquisa Nacional de Saúde 2013: percepção do estado de saúde, estilos de vida e doenças crônicas. Rio de Janeiro: IBGE; 2014. Disponível em: ftp://ftp. ibge.gov.br/PNS/2013/pns2013.pdf

22. Instituto Brasileiro de Geografia e Estatística. Pesquisa de orçamentos familiares 2008-2009: antropometria e estado nutricional de crianças, adolescentes e adultos no Brasil. Rio de Janeiro: IBGE; 2010. Disponível em: http://biblioteca.ibge.gov.br/visualizacao/livros/liv45419.pdf

23. World Health Organization. Obesity: preventing and managing the global epidemic. Report of a WHO Consultation on Obesity. Geneva: WHO; 2000.

24. Martins APB, Levy RB, Claro RM, Moubarac JC, Monteiro CA. Participação crescente de produtos ultraprocessados na dieta brasileira (1987-2009). Rev. Saúde Pública 2013; 47(4):656-65

25. Brasil. Ministério da Saúde. Guia alimentar para a população brasileira. Promovendo alimentação saudável. Brasília: Ministério da Saúde; 2006. 210 p. 
26. Pinheiro ARO. Reflexões sobre o processo histórico/político de construção de Lei Orgânica de Segurança Alimentar e Nutricional. Segurança Alimentar e Nutricional 2008; 15(2):1-15.

27. Cohen D, Farley TA. Eating as an automatic behavior. Prev Chronic Dis. 2008; 5(1):A23).

28. Mintz S, Du Bois C. The anthropology of food and eating. Annual Review of Anthropology 2002; 31:99-119.

Recebido: $12 / 1 / 2016$

Revisado: $20 / 7 / 2016$

Aceito: 30/8/2016 